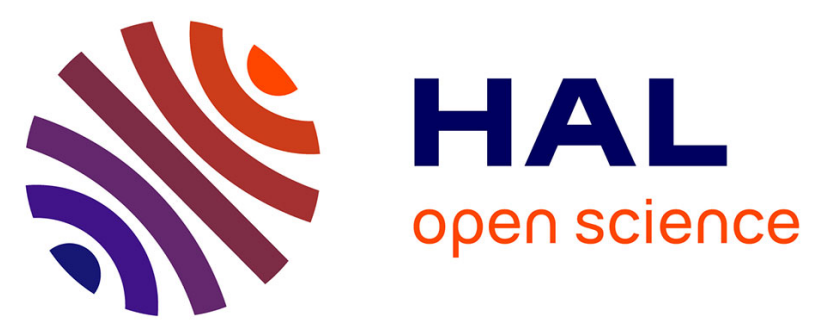

\title{
Evaluation of attitude estimation algorithms using absolute magnetic reference data: Methodology and results
}

Thomas Brunner, Sébastien Changey, Emmanuel Pecheur, Jean-Philippe Lauffenburger, Michel Basset

\section{To cite this version:}

Thomas Brunner, Sébastien Changey, Emmanuel Pecheur, Jean-Philippe Lauffenburger, Michel Basset. Evaluation of attitude estimation algorithms using absolute magnetic reference data: Methodology and results. Position, Location and Navigation Symposium - PLANS 2014, May 2014, Monterey, United States. 10.1109/PLANS.2014.6851378 . hal-01123475

\section{HAL Id: hal-01123475 \\ https://hal.science/hal-01123475}

Submitted on 4 Mar 2015

HAL is a multi-disciplinary open access archive for the deposit and dissemination of scientific research documents, whether they are published or not. The documents may come from teaching and research institutions in France or abroad, or from public or private research centers.
L'archive ouverte pluridisciplinaire HAL, est destinée au dépôt et à la diffusion de documents scientifiques de niveau recherche, publiés ou non, émanant des établissements d'enseignement et de recherche français ou étrangers, des laboratoires publics ou privés. 


\section{Evaluation of attitude estimation algorithms using absolute magnetic reference data: methodology and results}

\author{
Thomas Brunner, Sébastien Changey, \\ Emmanuel Pecheur \\ French-German Research Institute of Saint-Louis (ISL) \\ Guidance, Navigation and Control (GNC) department \\ Saint-Louis, France \\ e-mail: thomas.brunner@isl.eu
}

\author{
Jean-Philippe Lauffenburger, Michel Basset \\ Laboratoire MIPS -EA2332 \\ Université de Haute Alsace \\ Mulhouse, France \\ e-mail: jean-philippe.lauffenburger@uha.fr
}

\begin{abstract}
Location is a growing problem due, for instance, to the expansion of civil drones or intelligent vehicles. This expansion was made possible through the development of microelectromecanical systems (MEMS), cheap and miniaturized inertial sensors. In this context, this article is devoted to the development of a simulator which generates the sensor measurements, giving a specific trajectory, in order to validate and compare pose estimation algorithms. After validation of the simulator with real movements and measurements, four pose estimation algorithms from the literature are compared on different trajectories. All these algorithms use magnetic field sensors in addition of the classical inertial sensors. This comparison is led to select an algorithm for a future application with multiple IMUs.

Index Terms-IMU; Modelling; Magnetometers; Pose Estimation
\end{abstract}

\section{INTRODUCTION}

Dynamic location of objects is a predominant research topic in many fields (robotics [1], intelligent vehicles [2], UAVs [3], bio-logging [4], etc). A well-known method is the use of satellite positioning systems (or Global Navigation Satellite System (GNSS)), which directly provides the position of a mobile using satellite transmitted signals. On the opposite, the inertial method exploits proprioceptive sensors (accelerometers and gyroscopes) and measures the position changes of the considered mobile. This latter allows other applications where satellite signals are not always available. The association of proprioceptive sensors is called an Inertial Measurement Unit (IMU). When levels of integrity and high availability are required, an IMU and a GNSS device can be coupled, usually using observer-based algorithms.

For the French-German Research Institute of Saint-Louis (ISL), which conducts research in the field of science and technology of weaponry, location is a key point in mobile guidance [5]. For sensitive applications, the use of GNSS is not possible because of blurring. In addition, some applications are subject to high dynamics (a few thousand of $\mathrm{g}$ and several hundreds of Hertz), making use of gyros impossible until now. With the rapid development of the MEMS (Micro -Electro Mechanical Systems) technology, low cost sensors that can withstand high dynamic loads are now common. To get rid of the mobile model used in observers, MEMS rate gyroscopes are now added to obtain a classical IMU augmented with magnetometers. The idea is to develop estimation algorithms which update the absolute magnetic measurements in the body frame with the absolute knowledge of the Earth magnetic field.

This paper presents, in a first step, the modeling of a magnetometer-augmented IMU. A Matlab simulator based on the kinematic models of the sensors is proposed. To achieve this modeling, different characteristics are taken into account: the multiple reference frames (inertial frame, body frame, etc); the placement of the IMU which can be different from the center of the object; the modeling of the accelerometers, rate gyroscopes and magnetometers which also takes into account several sensor imperfections (bias, noise). Finally, this tool is designed to provide sensor measurements, from an input trajectory defined by the object orientation quaternion and its spatial position (see figure 1). Afterwards, the validation of the sensor model equations is presented. It consists in placing an IMU in a 3-axes table which has been developed at ISL. The absolute encoders of the electric engines are used to measure the $3 \mathrm{D}$ motion of the table.

In a second step, the paper presents the performance evaluation of 4 GPS-free orientation observers. The compared algorithms are all based on a magnetic sensor-augmented IMU. The most common method for orientation estimation is the well known Kalman Filter (KF) in its linear (LKF) or Extended (EKF) form [6]. A Complementary Observer (CO) [7] and a Gradient Descent Algorithm (GDA) [8] are also evaluated. These observers are tested on different trajectories. To evaluate these algorithms, the following points are observed: the precision, the execution time and the sensibility to accelerometric perturbation.

The outline of the paper is as follows: section II describes the magnetometer-augmented IMU modelling, section III shows the evaluation of the algorithms and section IV concludes the paper.

\section{Magnetometer-AugMented IMU MODELLING}

\section{A. Principle}

The simulator's aim is to analytically determine all the sensor measurements from the IMU according to a measured or an a priori known object trajectory. The principle of the simulator is shown in figure 1. From a trajectory describing the evolution of the spatial position $\left[s_{\mathrm{BL}}\right]^{\mathrm{L}}$ and the angular position (using the quaternion $\left\{q^{\mathrm{BL}}\right\}^{\mathrm{L}}$ ), the simulator determines 


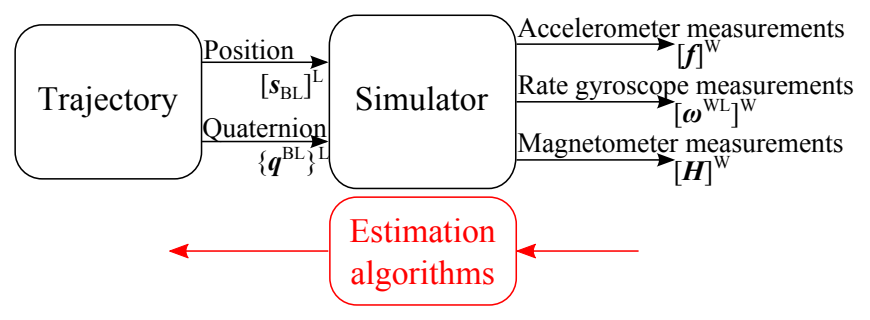

Fig. 1. Simulator principle.

equations of the accelerations, the rotational speeds and the magnetic measurements of the IMU sensors. Thus, the simulator performs an inverse modeling of the object dynamics. Its main purpose is the validation of motion estimation algorithms by comparing the input trajectory to the estimated one. This trajectory may correspond to a real or simulated movement to test the estimation algorithms in the desired conditions.

\section{B. Coordinate systems}

There are numerous reference frames which are employed in navigation ([9] and [10]). For the development of the simulator, a quick description of each used reference frame is given hereafter:

- The Earth frame (E) which is centered on the Earth, has the same axes than the Inertial frame (I) at $t=0$. Unlike (I) which is fixed, (E) is following the Earth rotation (constant speed $\omega^{\mathrm{EI}}$ ). The Earth, Inertial and Local frames are illustrated in figure 2.

- The Local frame (L) is fixed in (E). Its location is on the surface of the Earth and its position is described by 2 angles: the latitude $(\lambda)$ and the longitude $(l)$.

- The Body frame (B) is linked to the object orientation. Its orientation with respect to (L) is given by the classical Euler angles.

- The IMU frame (W) can be misaligned with the body frame. However, (W) is fixed with respect to (B) and is dependent of its location on the object.

Any axis of a frame is named by the lowercase of the frame name and a subscipt giving its number such that the 3 vectors of the frame (E) are then $e_{1}, e_{2}$ and $e_{3}$ (see figure 2).

\section{Sensor models}

This section describes the models of the sensors considered in the magnetometer-augmented IMU simulator. The sensor modeling considers no measurement imperfections. Bias and white noise are then added to these errorless variables. The final objective of this research is to provide pose observers taking into account the absolute magnetic reference data. That is why, in this section, a particular interest will be put on the magnetic field modeling and magnetometer measures estimation. The other equations were already detailed in [11].

1) Magnetometers modeling: A 3 axes magnetometer measures the direction and the intensity of the magnetic field around the sensor. If this magnetic field is not perturbed, it corresponds to the Earth magnetic field. The magnetometer

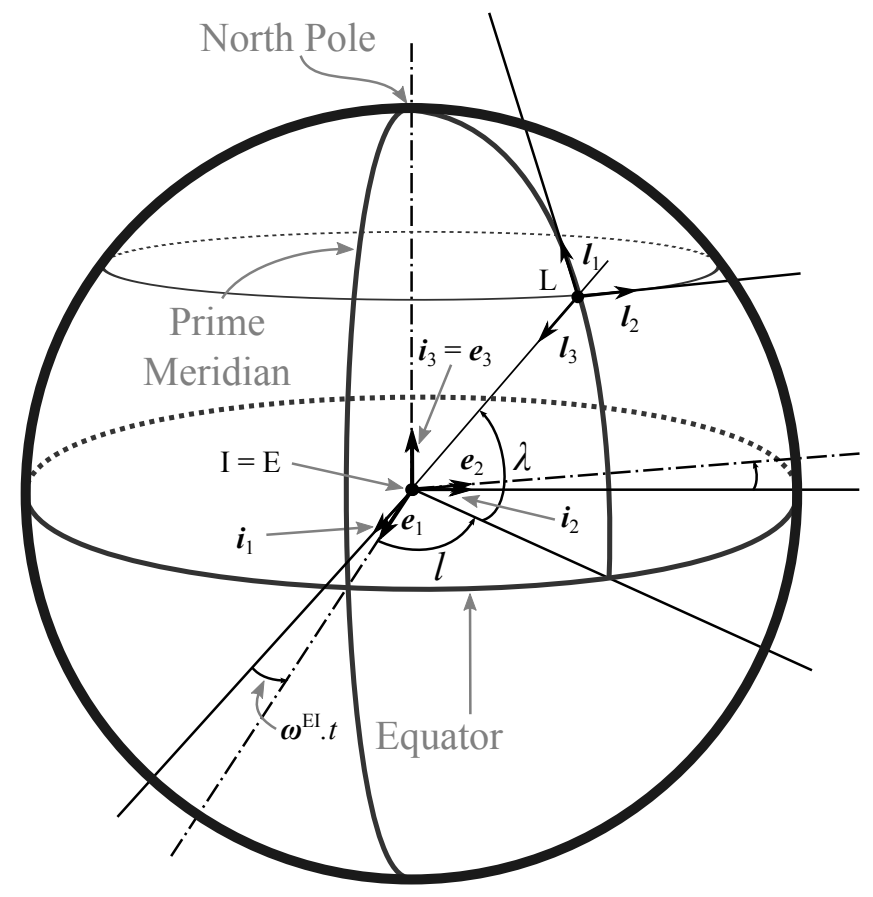

Fig. 2. Illustration of the different frames: the inertial frame (I), the earth frame (E) and the local frame (L).

measurements are the projection of this magnetic field in the frame $(\mathrm{W})$ :

$$
[h]^{\mathrm{W}}=[\mathbf{T}]^{\mathrm{WL}}[h]^{\mathrm{L}},
$$

with $[\mathbf{T}]^{\mathrm{WL}}$ the transformation matrix from frame (L) to frame (W). The Earth magnetic field is (in the Northern Hemisphere) directed to the North magnetic Pole and the inside of the Earth. The difference of position between the North magnetic and geographic poles leads to a non-zero component on the $l_{2}$ axis. The knowledge of the reference value of the magnetic field is then primordial for the heading correction of the different pose estimators. An Earth magnetic field model, called the World Magnetic Model [12], has been implemented in the simulator to provide the reference unperturbed magnetic value at the position of the object. This model allows also simulating any movement between the 2 polar circles. The value of $[h]^{\mathrm{L}}$ is given by this model.

This model was obtained by the interpolation of multiples measurement centers all on the Globe and is an empirical model. This is why it is only valid for 5 years and is constantly evolving. The implementation of this model also provides the value of the reference everywhere on the surface of the Earth. This allows simulations of trajectory everywhere but also simulations of long drive.

2) Rate gyroscopes modeling: A rate gyroscope gives the angular rate of the sensor with respect to the inertial frame [13] expressed in the sensors frame (W). These measurements can be determined from the quaternion and its derivative [14]:

$$
\left\{\dot{q}^{\mathrm{WI}}\right\}^{\mathrm{I}}=\frac{1}{2}\left\{q^{\mathrm{WI}}\right\}^{\mathrm{I}} \otimes\left\{\omega^{\mathrm{WI}}\right\}^{\mathrm{W}},
$$




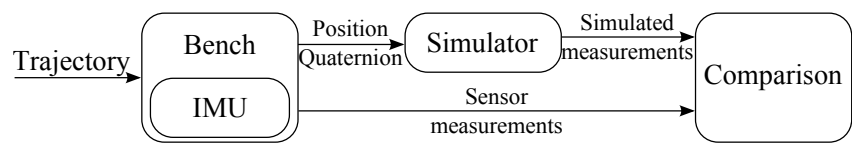

Fig. 3. Simulator test sketch with a real IMU.

with $\left\{q^{\mathrm{WI}}\right\}^{\mathrm{I}}$ the quaternion expressing the orientation of $(\mathrm{W})$ in $(\mathrm{I}),\left\{\dot{q}^{\mathrm{WI}}\right\}^{\mathrm{I}}$ its derivative with respect to time and $\left\{\omega^{\mathrm{WI}}\right\}^{\mathrm{W}}$ the pure quaternion formed from the rotational speed vector of (W) with respect to (I). From (2), it is straightforward to obtain $\left\{\omega^{\mathrm{WI}}\right\}^{\mathrm{W}}$.

3) Accelerometers modeling: An accelerometer measures the specific force applied along its principle axis (in frame W). Specific force is defined by the sum of all ungravitational forces applied on the object divided by the object mass. It is then the absolute acceleration of the object $\left(a_{\mathrm{W}}^{\mathrm{I}}\right)$ minus the gravitational field, such that:

$$
[f]^{\mathrm{W}}=\left[a_{\mathrm{W}}^{\mathrm{I}}\right]^{\mathrm{W}}-\left[g_{\mathrm{f}}\right]^{\mathrm{W}} .
$$

As a reminder, the gravitational field is expressed as [10]:

$$
g_{\mathrm{f}}=g+\gamma_{e}=-G M \frac{s_{\mathrm{WE}}}{\left\|s_{\mathrm{WE}}\right\|^{3}}-\omega^{\mathrm{EI}} \wedge\left(\omega^{\mathrm{EI}} \wedge s_{\mathrm{WE}}\right),
$$

where $s_{\mathrm{WE}}$ is the position of the sensors with respect to (E), $G$ is the gravitational constant and $M$ is the mass of the Earth.

The objective is then to determine the expression of $[f]^{\mathrm{W}}$ from the evolution of the object position $\left[s_{\mathrm{BL}}\right]^{\mathrm{L}}$ which is an input of the simulator. However, the sensors are not positioned at the center of the object. Consequently, deriving the position of the object will not lead to the acceleration of the IMU but to the object one. After transforming the input position $\left[s_{\mathrm{BL}}\right]^{\mathrm{L}}$ in frame (E), the equation of the total acceleration applied on the IMU is given by ${ }^{1}[10]$ :

$$
\begin{aligned}
a_{\mathrm{W}}^{\mathrm{I}}= & a_{\mathrm{B}}^{\mathrm{E}}+\left.\frac{d \omega^{\mathrm{BE}}}{d t}\right|_{\mathrm{B}} \wedge s_{\mathrm{WB}}+\omega^{\mathrm{BE}} \wedge\left(\omega^{\mathrm{BE}} \wedge s_{\mathrm{WB}}\right) \\
& +\omega^{\mathrm{EI}} \wedge\left(2\left(v_{\mathrm{B}}^{\mathrm{E}}+\omega^{\mathrm{BE}} \wedge s_{\mathrm{WB}}\right)+\omega^{\mathrm{EI}} \wedge s_{\mathrm{WI}}\right) .
\end{aligned}
$$

Finally, the specific force can be obtained by subtracting (4) and (5).

\section{Validation}

After implementing the latter equations in Matlab, a validation process is performed. For that, the sketch of figure 3 is followed. A comparison between the simulated measurements and the real ones is done. To achieve this, a 3D rotation table from ISL was used (see figure 4). The IMU is fixed at a known position and rotations are performed. The table encoders are used to measure the Euler angles of the performed movement. These encoders are known to provide the orientation with a very good precision $\left(<0.1^{\circ}\right)$. Here, the mobile part of the table is considered as the body, so only the orientation of the body is evolving, the position of the center of the body is static.

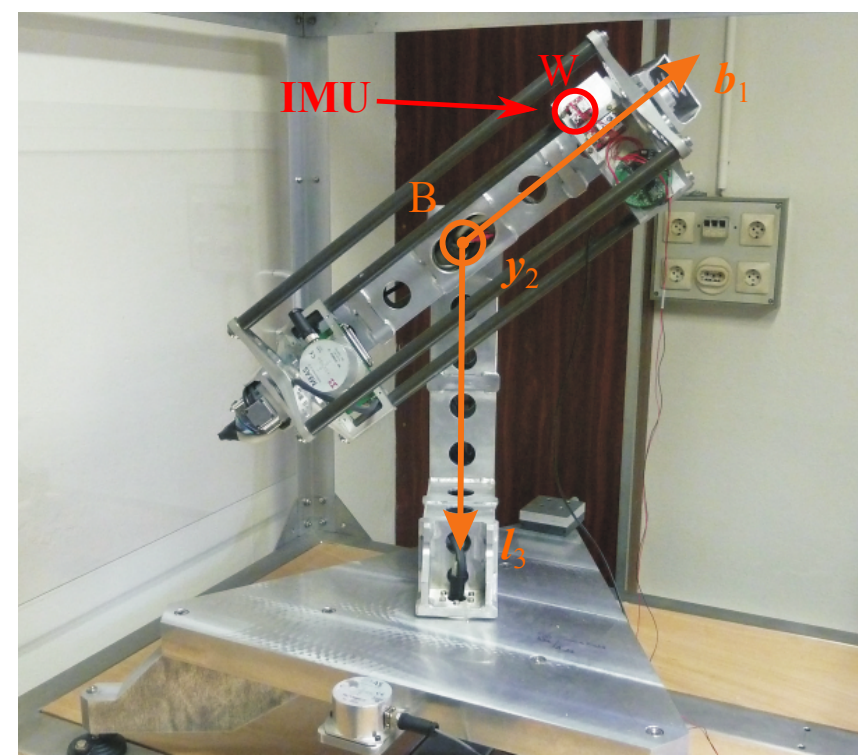

Fig. 4. Test bench: 3-axes table.
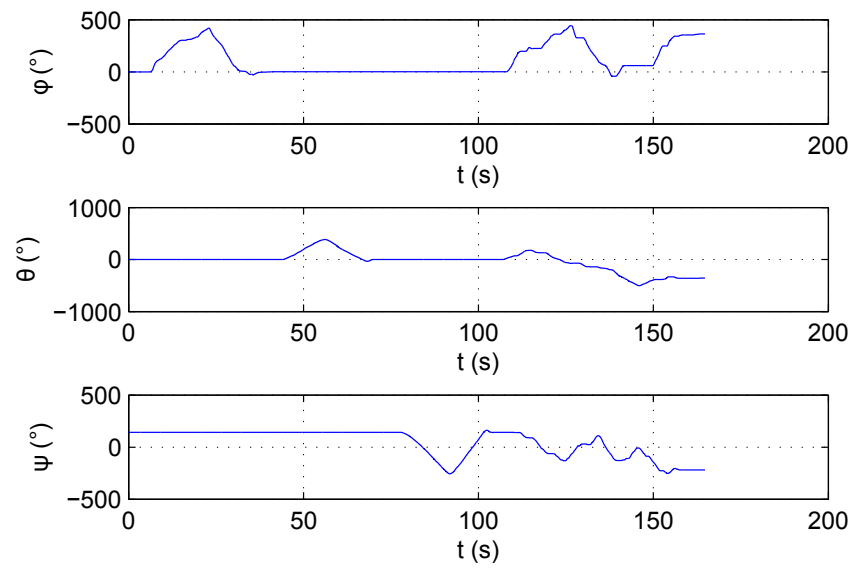

Fig. 5. Measured angles during the validation test.

The performed movement is characterized by the Euler angles illustrated in figure 5. This movement is divided in 2 parts. The first part, up to $t=100 \mathrm{~s}$, is composed of 3 pure rotations around each axis separately, the second part of the movement is an arbitrary rotation around the 3 axes simultaneously. The measurement comparisons of each type of sensors are shown in figure 6,7 and 8 . It can be seen that the simulated measurements are very close to the real ones.

The overall performance of the simulator is shown in table I. The errors of the accelerometer measurements are $2.5 \%$ at maximum and the standard deviation is quite low, characteristic of a little bias mistrimmed on the $w_{3}$ axis. The rate gyroscopes display a mean error below $0.2 \%$, it represents around $1 \%$. The magnetometers also show errors less than $3 \%$.

\footnotetext{
${ }^{1}$ For the sake of clarity, it's assumed that all variables are expressed in the same coordinate system, the brackets []$^{\mathrm{X}}$ are then forsaken.
} 

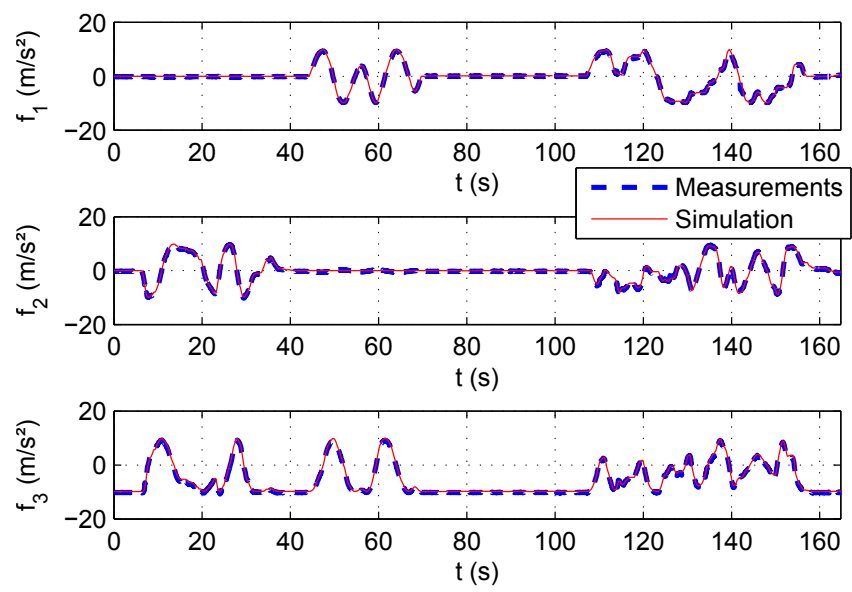

Fig. 6. Comparison of the accelerometer measurements.
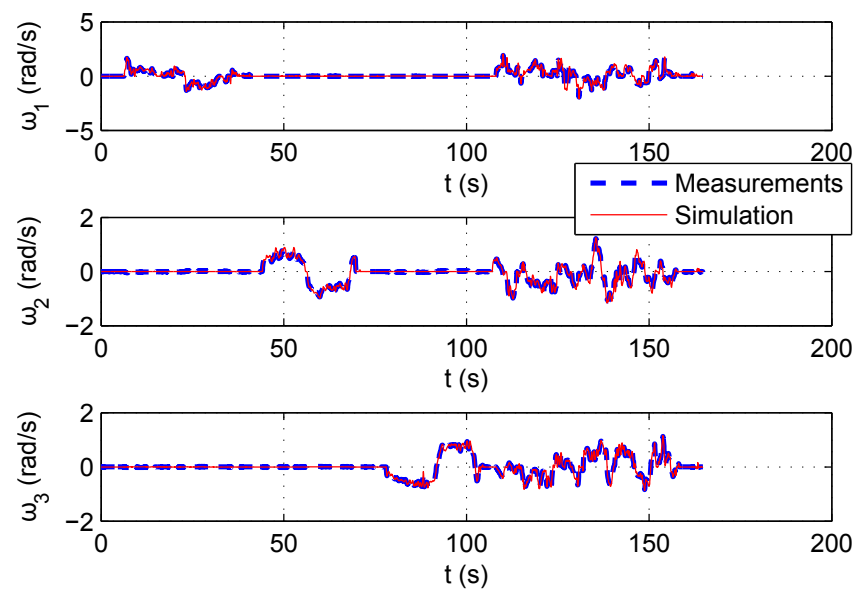

Fig. 7. Comparison of the rate gyroscope measurements.
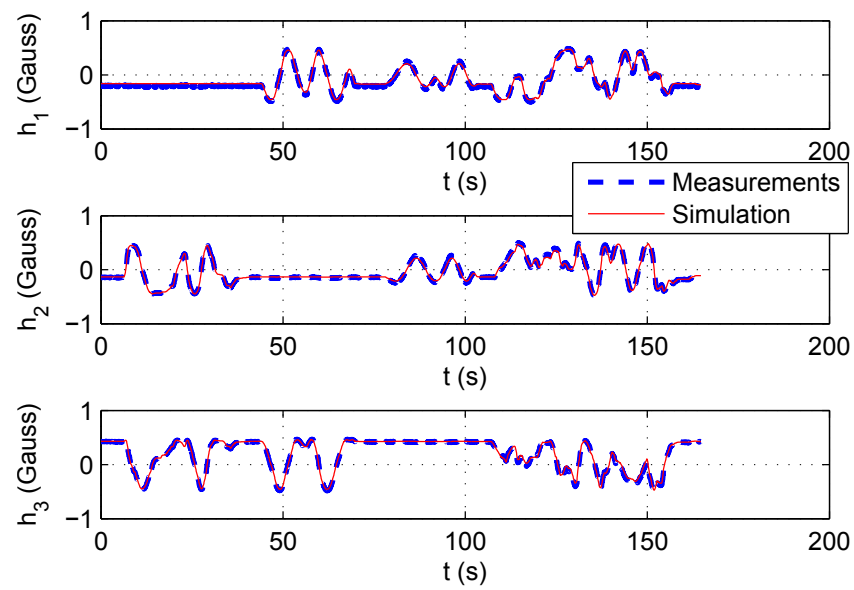

Fig. 8. Comparison of the magnetometer measurements.

\begin{tabular}{|c|c|c|c|}
\hline Sensors & Component & Error & Std \\
\hline \multirow{3}{*}{ Accelerometers } & $f_{1}$ & 0.91 & 0.95 \\
\cline { 2 - 4 } & $f_{2}$ & 1.03 & 0.15 \\
\cline { 2 - 4 } & $f_{3}$ & 2.22 & 0.11 \\
\hline \multirow{3}{*}{ Rate gyroscopes } & $\omega_{1}$ & 0.15 & 0.82 \\
\cline { 2 - 4 } & $\omega_{2}$ & 0.15 & 1.50 \\
\cline { 2 - 4 } & $\omega_{3}$ & 0.11 & 1.05 \\
\hline \multirow{3}{*}{ Magnetometers } & $h_{1}$ & 2.67 & 2.54 \\
\cline { 2 - 4 } & $h_{2}$ & 0.13 & 2.03 \\
\cline { 2 - 4 } & $h_{3}$ & 1.18 & 2.10 \\
\hline
\end{tabular}

TABLE I

ERRORS AND STANDARD DEVIATIONS OF THE ERRORS FROM THE Simulation VAlidation. All Data aRe GiVEN IN PERCENTAGE OF THE FULL SCALE MEASURED.

\section{Algorithm evaluation}

Now that the simulator is validated, it is possible to use it to evaluate and compare different pose estimation algorithms.

\section{A. Algorithm description}

To estimate the position of an object thanks to inertial sensors, multiples algorithms exist. Usually, these observers are validated on private data using specific metrics as well as test conditions. In this context, four algorithms will be quickly described $^{2}$ and tested in iso-conditions using the measurement simulator.

A Complementary Observer (CO) [7], a Gradient Descent Algorithm (GDA) [8], a classic Extended Kalman Filter (EKF) [6] and a Linear Kalman Filter (LKF) [6] have been implemented. All of them are using the measurements from the 3 types of sensors. Also, all are based on the quaternion evolution equation (2). This equation can be rewritten in terms of matrices and vectors instead of quaternions:

$$
\left[\dot{q}^{\mathrm{WI}}\right]^{\mathrm{I}}=\frac{1}{2}\left[\omega^{\mathrm{WI}}\right]_{\times}^{\mathrm{W}}\left[q^{\mathrm{WI}}\right]^{\mathrm{I}}
$$

where a matrix noted $[r]_{\times}$is extracted from a quaternion $\{r\}$ such that:

$$
[r]_{\times}=\left[\begin{array}{cccc}
r_{0} & -r_{1} & -r_{2} & -r_{3} \\
r_{1} & r_{0} & r_{3} & -r_{2} \\
r_{2} & -r_{3} & r_{0} & r_{1} \\
r_{3} & r_{2} & -r_{1} & r_{0}
\end{array}\right] .
$$

Another common point is the use of the accelerometer and magnetometer measurements to correct the errors of the gyroscopes like the biases or the random walk coming from the integration of the gyroscopes noises.

In the following, $\omega$ will refer to the rotational speed vector or quaternion, $d$ to a known reference vector, $s$ to its measurement and $q$ will be the quaternion expressing the orientation of (W) with respect to (L) expressed in (L).

\footnotetext{
${ }^{2}$ For more information about each algorithm, please refer to the cited papers.
} 
1) Complementary Observer: Fourati et al. introduced the Complementary Observer (CO) [7] in order to estimate the motion of animals. It uses the motion kinematic model described by equation (2). It is a two-layer filter architecture: a Levenberg Marquardt algorithm (LMA) processes the magnetometer and accelerometer measurements to produce a quaternion. The LMA is employed to solve the Wahba problem [15]. The quaternion from the LMA, noted $q_{m}$, and the one from the rate gyroscopes measurements, noted $q_{\omega}$ are combined, through complementary filters, respectively a low pass and a high pass filter, in the $\mathrm{CO}$ to obtain the output of the filter $(q)$ :

$$
q=F_{1} q_{m}+F_{2} q_{\omega}
$$

with

$$
F_{1}=\frac{K}{s+K} \quad \text { and } \quad F_{2}=\frac{s}{s+K},
$$

with $K$ the tunable matrix. Note also that $F_{1}+F_{2}=1 . F_{1}$ filters the noise and reduces the effect of accelerations on the attitude estimation process where $F_{2}$ uses the precision of the rate gyros in high frequency and removes the bias of the sensors which is in the low frequency domain. The gain $K$ is computed through these algorithm. This approach is well adapted to separate the high and low dynamics using this two filters. However, its implementation needs the tuning of the gain matrix $K$ for which the authors do not give a specific procedure.

2) Gradient Descent Algorithm: The second pose estimator uses the Gradient Descent Algorithm (GDA). Madgwick et al. introduced the GDA [8] in order to replace the traditional EKF in every low cost IMU by a computationally more efficient algorithm. It uses the acceleration and magnetic data in an analytically derived and optimized GDA to compute the direction of the gyroscope measurement error.

This optimization solution minimizes the function (10) using the GDA of (11).

$$
\begin{gathered}
f(q, d, s)=\tilde{q} d q-s, \\
q_{\nabla, t}=q_{\mathrm{est}, t}-\mu \frac{\nabla f}{\|\nabla f\|},
\end{gathered}
$$

with

$$
\nabla f=\mathbf{J}(q, d) f(q, d, s),
$$

with $\mathbf{J}$ the jacobian matrix of $f$ with resprect to $q$ and $d$.

After using the gravity and magnetic measurement to compute $q_{\nabla, t}$, the next step is the fusion between $q_{\nabla, t}$ and $q_{\omega, t}$ coming from the integration of the rate gyroscope measurements. To achieve this, the following equation is used:

$$
q_{\mathrm{est}, t}=\gamma_{t} q_{\nabla, t}+\left(1-\gamma_{t}\right) q_{\omega, t} .
$$

In fine, after some simplifications, the equations of fusion are:

$$
\begin{gathered}
q_{\mathrm{est}, t}=q_{\mathrm{est}, t-1}+\dot{q}_{\mathrm{est}, t} \Delta t, \\
\dot{q}_{\mathrm{est}, t}=\dot{q}_{\omega, t}-\beta \frac{\nabla f}{\|\nabla f\|},
\end{gathered}
$$

with $\beta$ the divergence rate of $q_{\omega, t}$. To select its value, the paper suggests to use $\beta=\sqrt{\frac{3}{4}} \omega_{\max }$, where $\omega_{\max }$ defines the maximum rotational speed of the device. As it is the only parameter to tune, the algorithm is easy to use but the tuning phase is not straightforward: if $\beta$ is too important, some oscillations are seen during static movements, if $\beta$ is too low, the algorithm is not able to follow the reference during high rotational speed movement.

3) Extended Kalman Filter: A very common algorithm is the Extended Kalman Filter as in [6]. The general EKF method is derived from equation (2). The state vector contains the quaternion elements $q$ and in general is augmented with the rate gyroscope bias $b$. The evolution equation of the EKF (in continuous time) is then:

$$
\left[\begin{array}{c}
\dot{q} \\
\dot{b}
\end{array}\right]=\left[\begin{array}{cc}
{[\omega-b]_{\times}} & \mathbf{0} \\
\mathbf{0} & \mathbf{0}
\end{array}\right]\left[\begin{array}{l}
q \\
b
\end{array}\right],
$$

where $b$ is the vector of the rate gyroscope bias and $\omega$ is the vector of the rate gyroscope measurement.

Usually, the measurements are the magnetic field and the gravity projection in the body frame. The measurement equation for one reference vector $s$ is:

$$
s=\tilde{q} d q,
$$

which is nonlinear with respect to the state $q$. After linearization, the measurement part of the evolution model is:

$$
Y=\mathbf{J}(X) X,
$$

with $X$ the state vector, $Y$ the measurement vector and $\mathbf{J}(X)$ the Jacobian matrix obtained by linearizing (17) for the gravity and the Earth magnetic fields.

4) Linear Kalman Filter: Chouckroun et al. introduced the Linear Kalman Filter (LKF) [6] in order to get rid of the linearization part in the EKF. The LKF is using the same evolution model than the previously described EKF. The difference lays in the measurement equation, where the non-linearity appeared. The idea is to keep the linearity by transforming (17):

$$
q s=d q,
$$

in

$$
q s-d q=0,
$$

which can be expressed in terms of matrices and vectors.

$$
\mathbf{H} q=0,
$$

with $\mathbf{H}$ the new measurement matrix.

\section{B. Evaluation conditions}

To evaluate the algorithms, three trajectories are designed. They are planed with a growing difficulty: the first one is a slow rotation motion with no displacement, to verify that all estimation algorithms work in ideal conditions. The orientation evolution is illustrated in figure 9. The second trajectory 

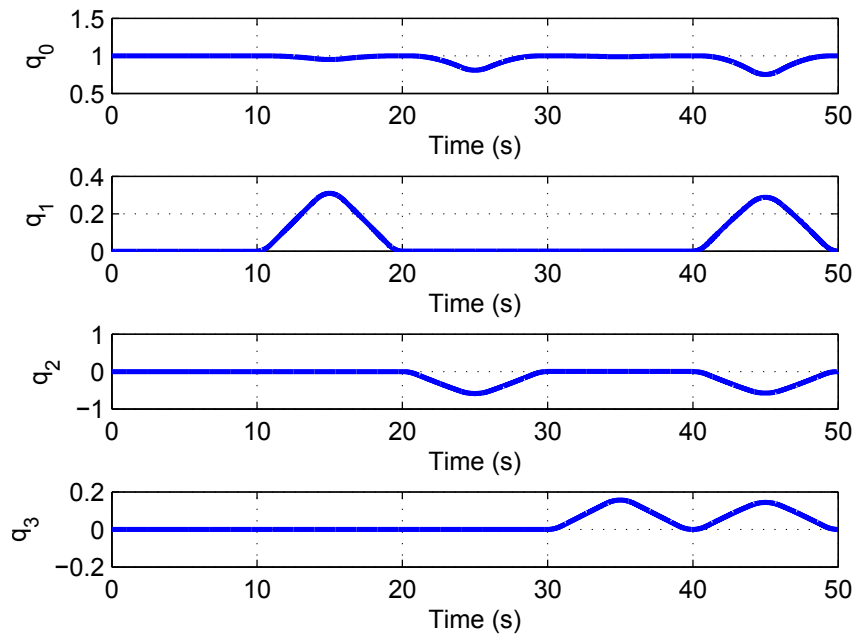

Fig. 9. Evolution of the quaternion describing the orientation of the first trajectory.
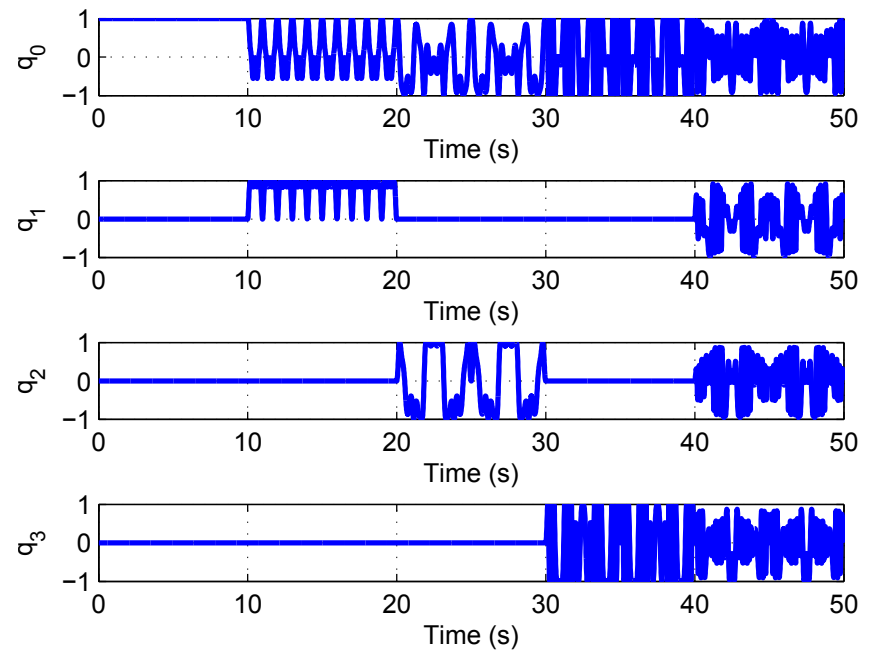

Fig. 10. Evolution of the quaternion describing the orientation of the second trajectory.

is also an only rotational movement but with much higher speed and bandwidth, allowing putting the algorithms under more constraints (see figure 10). The third trajectory is a translation-only motion to evaluate the effect of acceleration on the orientation estimation. The evolution of the position is illustrated in figure 11.

To simulate a real IMU, noises are added to the measurements. The power of the noise is taken considering an IMU datasheet [16]. Biases are supposed to be compensated through the calibration process.

\section{Results}

After the use of the first trajectory to tune each parameters of the different algorithms, all of them show errors lower than 0.03 on each quaternion component which represent less than $3^{\circ}$ on each angles (see figure 12). The GDA displays a constant bias of $1.4^{\circ}$ on $\psi$. This is due to the fact that this algorithm

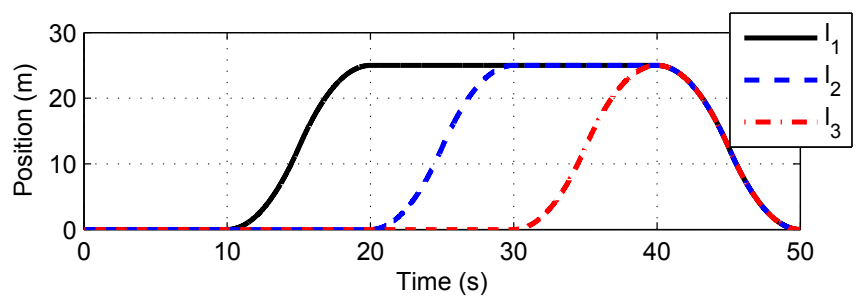

Fig. 11. Evolution of the position during the third trajectory.
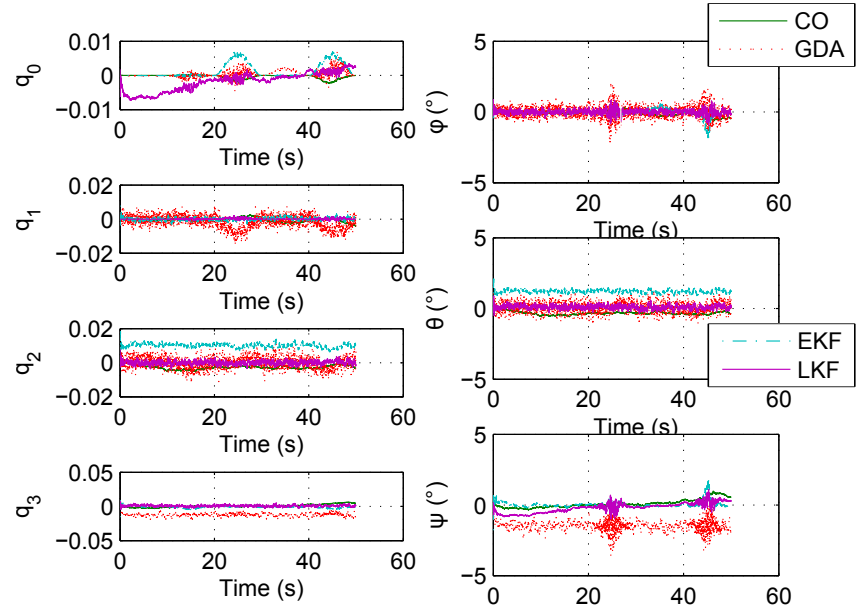

Fig. 12. Error on the quaternion components and the Euler angles on trajectory one (figure 9).

is considering the magnetic and geographic North poles to be at the same position.

The second trajectory allows testing the pose algorithms in higher dynamics. Table II helps to synthesize the results. Even if the mean errors are very low for each algorithm, the standard deviations are not equivalent. The LKF is the most accurate, closely followed by the EKF. The GDA is relatively close to the reference but the $\mathrm{CO}$ is very far from the others.

The third trajectory shows the influence of acceleration on the orientation estimation. The impacts of the acceleration on the Euler angles are plotted in figure 13. It can be seen that an acceleration, between $10 \mathrm{~s}$ and $20 \mathrm{~s}$, on the first axis $\left(l_{1}\right)$ leads to an error on $\theta$, but an acceleration on the second axis

\begin{tabular}{|c|c|c|c|c|c|}
\cline { 3 - 6 } \multicolumn{2}{c|}{} & CO & GDA & EKF & LKF \\
\hline \multirow{2}{*}{$e_{q_{0}}$} & Mean & -0.023 & 0.004 & 0.001 & 0.000 \\
\cline { 2 - 6 } & Std & 0.351 & 0.183 & 0.061 & 0.048 \\
\hline \multirow{2}{*}{$e_{q_{1}}$} & Mean & -0.002 & 0.004 & 0.002 & 0.001 \\
\cline { 2 - 6 } & Std & 0.141 & 0.128 & 0.027 & 0.021 \\
\hline \multirow{2}{*}{$e_{q_{2}}$} & Mean & 0.005 & 0.003 & 0.003 & 0.000 \\
\cline { 2 - 6 } & Std & 0.213 & 0.143 & 0.024 & 0.012 \\
\hline \multirow{2}{*}{$e_{q_{3}}$} & Mean & -0.006 & 0.001 & -0.002 & 0.001 \\
\cline { 2 - 6 } & Std & 0.341 & 0.180 & 0.062 & 0.048 \\
\hline
\end{tabular}

TABLE II

ERRORS AND STANDARD DEVIATIONS OF THE ERRORS FROM THE SECOND TRAJECTORY (FIGURE 10) (ABSOLUTE VALUES). 

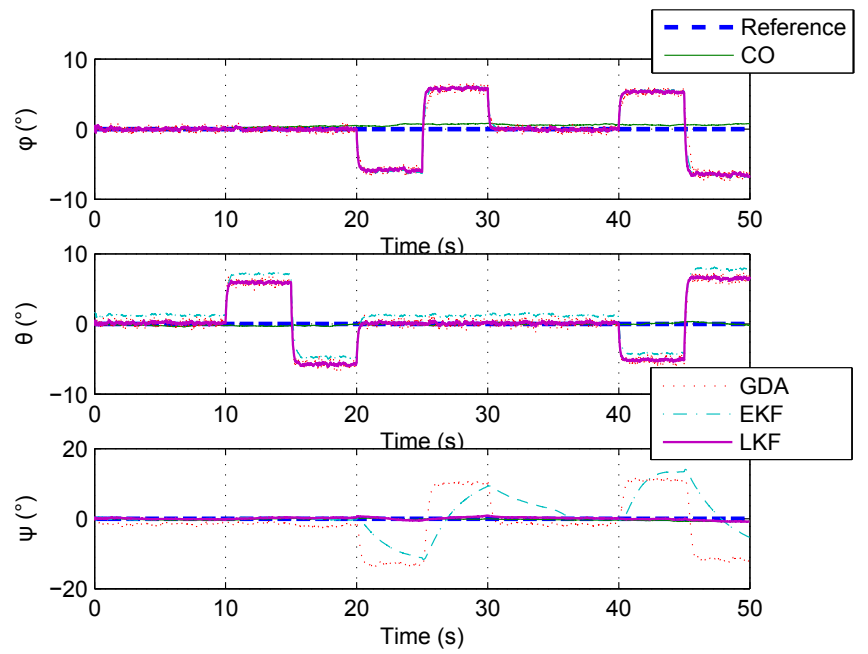

Fig. 13. Influence of the acceleration on the estimation of the Euler angles from the third trajectory (figure 11).

$\left(l_{2}\right)$, between $20 s$ and $30 s$, leads to an error on both $\psi$ and $\phi$. Except the CO approach, all the algorithms are affected by the perturbation.

Concerning the execution time, the results are approximately the same for each trajectory. For 5000 iterations (one trajectory), the excution times are respectively of $1 \mathrm{~s}, 0.5 \mathrm{~s}, 0.7 \mathrm{~s}$ and 1.6s for the CO, GDA, EKF and LKF. This represents respectively $0.2 \mathrm{~ms}, 0.1 \mathrm{~ms}, 0.14 \mathrm{~ms}$ and $0.32 \mathrm{~ms}$ per iteration.

These execution times are given for a comparison between each algorithm, not an exact value when embedded. Indeed, these results depend on the implementation and the computer executing the simulations. All of the algorithms are coded on Matlab and tested on a standard i7 quad core computer.

The LKF seems then to be the slowest algorithm but also the most accurate when there is no perturbation. As the $\mathrm{CO}$ is developed for animal motion estimation, it can be seen that there are some problems in very high dynamics but is robust to acceleration perturbation. The GDA replaces the EKF in classical low cost IMU is a bit less accurate than the EKF but more computationally efficient.

\section{CONCLUSION}

This article has presented the development of a simulator which provides the measurement of an IMU augmented with magnetometers from a known trajectory. The used sensors are 3D rate gyroscopes, accelerometers and magnetometers. The modeling is based on the quaternion representation of the orientations avoiding singularities from the Euler angles.

The results obtained with the simulator are compared to the calibrated measurements of a real IMU. The validation is made thanks to a precise 3D rotational table. The different results show the precision of the simulator, validating the equations.

This simulator is then used to evaluate 4 pose estimation algorithms: a Complementary Observer, a Gradient Descent Algorithm, an Extended Kalman Filter and a Linear Kalman
Filter. These algorithms are tested on different trajectories and are evaluated according to 3 parameters: the precision of output quaternion, the execution time and the accelerometric perturbation. The LKF is the most accurate but also the slowest. The GDA and the EKF have a low execution time and are relatively accurat. The only algorithm unperturbed from lateral acceleration is the $\mathrm{CO}$, unlikely, it is the less accurate.

Some investigations can be made in the future to understand why the execution time of the LKF is higher than for the EKF. An EKF seems to be a good compromise between accuracy and execution time but each algorithm has its drawbacks. An investigation may be made on why the $\mathrm{CO}$ is not perturbed by the lateral accelerations.

The simulator can be upgraded taking account of the sensors' bandwith or non stationnary biases.

\section{ACKNOWLEDGMENT}

The authors would like to thank the Alsace Region and the French-German Research Institut of Saint-Louis for the founding of this work.

\section{REFERENCES}

[1] S. Kucuk and Z. Bingul, "Robot kinematics: Forward and inverse kinematics," pp. 117-148, 2006.

[2] Y. Yang, "Spacecraft attitude determination and control: Quaternion based method," Annual Reviews in Control, vol. 36, pp. 198-219, Dec. 2012.

[3] M. Euston, P. Coote, R. Mahony, J. Kim, and T. Hamel, "A complementary filter for attitude estimation of a fixed-wing uav," pp. 340-345, 2008.

[4] H. Fourati, N. Manamanni, L. Afilal, and Y. Handrich, "A nonlinear filtering approach for the attitude and dynamic body acceleration estimation based on inertial and magnetic sensors: Bio-logging application," IEEE Sensors Journal, vol. 11, pp. 233-244, Jan. 2011.

[5] S. Changey, E. Pecheur, L. Bernard, E. Sommer, P. Wey, and C. Berner, "Real time estimation of projectile roll angle using magnetometers: Inflight experimental validation," in 2012 Ieee/Ion Position Location and Navigation Symposium (plans), pp. 371-376, 2012.

[6] D. Choukroun, I. Y. Bar-Itzhack, and Y. Oshman, "A novel quaternion kalman filter," IEEE Transactions on Aerospace and Electronic Systems, vol. 42, pp. 174-190, Jan. 2006.

[7] H. Fourati, N. Manamanni, L. Afilal, and Y. Handrich, "Complementary observer for body segments motion capturing by inertial and magnetic sensors," IEEE/ASME Transactions on Mechatronics, Feb. 2013.

[8] S. Madgwick, A. J. L. Harrison, and R. Vaidyanathan, "Estimation of imu and marg orientation using a gradient descent algorithm," pp. 1-7, 2011.

[9] V. Sircoulomb, Etude des concepts de filtrage robuste aux méconnaissances de modèle et aux pertes de mesures. $\mathrm{PhD}$ thesis, Nancy-Université, Nancy, France, Dec. 2008.

[10] P. H. Zipfel, Modeling and Simulation of Aerospace Vehicle Dynamics. AIAA, second ed., 2000.

[11] T. Brunner, S. Changey, J.-P. Lauffenburger, M. Basset, and E. Pecheur, "Modélisation d'une unité de mesure inertielle augmentée de capteurs magnétiques à référence absolue," (Strasbourg), p. 6, 2013.

[12] S. Maus, S. Macmillan, S. McLean, B. Hamilton, A. Thomson, M. Nair, and C. Rollins, The US/UK world magnetic model for 2010-2015. NOAA National Geophysical Data Center, 2010.

[13] J. Radix, Systèmes Inertiels à Composants Liés "Strap-Down". Sup'Aero, Cépaduès-Editions, 1991.

[14] R. Goldman, "Understanding quaternions," Graphical Models, vol. 73, pp. 21-49, Mar. 2011.

[15] G. Wahba, "A least squares estimate of satellite attitude," SIAM Review, vol. 8, pp. 384-386, July 1966

[16] S. Systems, "Ig-500n gps aided ahrs user manual," 2008. 